\title{
IMPORTANCE OF NUTRITION ASSESSMENT IN CRITICALLY ILL PATIENTS
}

\section{Suganya ${ }^{1 *}$, Vasantha Esther Rani}

${ }^{1}$ Madurai KamarajUniversity, Madurai, India

${ }^{2}$ Head of the Home Science Department, Fatima College, Madurai, India

Received - July 18, 2020; Revision - September 17, 2020; Accepted - November 02, 2020

Available Online - March 25, 2021

DOI: http://dx.doi.org/10.18006/2021.9(Spl-1-GCSGD_2020).S55.S61

\section{KEYWORDS \\ Intensive Care Unit \\ Subjective Global Assessment \\ Acute Physiology and Chronic \\ Health Evaluation \\ Body Mass Index}

\begin{abstract}
A timely assessment of nutritional status in critically ill patients is crucial. This was an observational prospective hospital study carried out for 3 months from December 2019 to February 2020 in an ICU in a multispecialty hospital in Madurai, Tamilnadu, India. All adult patients with $\geq 72$ hours of ICU-LOS were included and their nutritional assessment was completed by SGA (Subjective Global Assessment) forms within 24 hours of ICU admission. Data collected regarding variables such as Age, Nutritional status, APACHE IV, SOFA score, presence of Comorbidities. All patients were studied about their duration of stay in ICU and hospital, Predicted Mortality rate, \& discharge outcome.120 patients were involved in the study in the age 62. $2 \pm 14$.years, among this $55.3 \%$ were males and rest $44.7 \%$ were females. Their complete nutritional assessment revealed that $50 \%, 43 \%$, and $17 \%$ were well nourished, moderately malnourished, severely malnourished respectively. A strong correlation was observed between nutritional status, morbidity, and their prolonged stay in ICU. Mean ICU \& hospital mortality percentage was $37 \%, 45 \%$ respectively. The average duration of stay in the ICU and hospital was 13, 20 days respectively. Participants anthropometric data decreased considerably (mean weight, MUAC, calf circumference) during ICU discharge day. BMI comparison of admission and discharge didn't show significant change. Timely nutrition intervention and medical nutrition therapy reduce the duration of patient stay in ICU and hospital and improved patient outcomes. The disease severity scoring system can be used as guidance for objective assessment of disease outcomes and estimation of the chance of recovery.
\end{abstract}

* Corresponding author

E-mail: suganya101973@gmail.com, suganya_v@apollohospitals.com(V. Suganya)

Peer review under responsibility of Journal of Experimental Biology and Agricultural Sciences.

Production and Hosting by Horizon Publisher India [HPI] (http://www.horizonpublisherindia.in/).

All rights reserved.
All the articles published by Journal of Experimental Biology and Agricultural Sciences are licensed under a Creative Commons Attribution-NonCommercial 4.0 International License Based on a work at www.jebas.org.

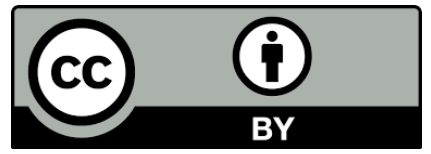




\section{Introduction}

Malnutrition is a global health problem in hospitalized patients, especially within the critical care setting. Especially for critically ill patients, malnutrition is noted as a major consequence of hospitalisation. Hyper metabolism in patients increases nutrient requirements but the patient's energy and protein intake were considerably less leading to malnutrition. Many research with critically ill patients showed that malnutrition before admission to the ICU leads to the diminished status of macro and micronutrients in their bodies. Nutritional Assessment on admission is the first and foremost step in a nutritional intervention which will help in reducing the duration of ICU and hospital stay and appropriate nutrition therapy prevent complications and infections thereby mortality risk will be reduced.

In critically ill patients, SIRS (systemic inflammation response syndrome) impacts a catabolic state that increases metabolic demand and accelerates the development of malnutrition (Heyland et al., 2011; Blaser et al., 2017). This study was conducted to assess the nutritional status of critically ill patients on ICU admission and discharge day through various clinical indicators and to highlight the importance of nutritional assessment for critically ill patients. Further, this study also correlated the mortality prediction score APACHE IV, SOFA scores on admission with patient's duration of stay in ICU and hospital along with patient outcome (Hopkins et al., 2017). The SOFA score is considered as an important tool in defining both the clinical condition of the individual patient and the response to therapies in the context of clinical trials (Simon Lambden et al., 2019). ICU patients were assessed with various clinical indicators to categorise their nutritional status. The anthropometric indices include BMI (body mass index), MUAC (mid upper arm circumference), and calf circumference. The body composition markers and biochemical investigations comprise visceral protein, total protein, albumin, and lymphocyte. A clinical questionnaire called SGA (Subjective global assessment) was applied in the study to analyse the participant's level of nutritional status. The SGA form widely accepted tool for identifying hospitalised patient's nutritional status including critically ill patients (Hopkins et al., 2017). Subjective global assessment (SGA) is an inexpensive, and quick method that can be conducted at the bedside. It is a reliable tool for inferring outcomes in critically ill patients (Herridge et al., 2016; McClave et al., 2016; Mehta et al.,2018).

\section{Materials and Methods}

\subsection{Study Design}

This was a hospital based prospective study duration, which was conducted for 3 months from December 2019 to February 2020, in a multidisciplinary ICU with a bed capacity of 21 beds in India,
Tamilnadu, Madurai. All adult patients admitted in ICU with $\geq 72$ hours of ICU-LOS were included and their nutritional assessment was completed by SGA (Subjective Global Assessment) forms. This process was completed soon after their ICU admission (within 24 hours). Reassessment was done once in seven days and during discharge and this was utilized for monitoring and evaluation of patient nutritional status.

\subsection{Study Population}

The sample size for the study was derived as 120 critically ill patients. The formula used for the study was $\mathrm{n}=\mathrm{Z} 2(\mathrm{SD} 2 / \mathrm{d} 2)$, taking a value of $Z=1.96$ with $95 \%$ confidence intervals, and $d=$ 0.7 as the margin of error in estimating the mean or effect size. A total of 325 patients admitted to the multidisciplinary ICU were screened but only 120 patients were in the eligibility criteria. The inclusion criteria considered as 18 years of age, adult patients with $\geq 72$ hours of ICU-LOS were included and other patients received from other hospitals ICU'S were excluded from the study. Approval obtained from Clinical Research and Institutional Ethics Committee of multispecialty hospital IEC application number ASH/ACAD-004/12-19, as per the requirement of the ethics committee informed consent was obtained from the patient's caretaker who admitted the patient.

\subsection{Data Collection}

Participant's unique ID number noted and demographic and clinical details collected along with, admission diagnosis, age, sex, category of admission - medical/surgical were noted within 24 hours of ICU admission. In this study mortality risk assessment score namely APACHE IV Score (Acute Physiology and Chronic Health Evaluation) (Chakravarty et al., 2013; Lew et al., 2017) was employed, SOFA (Sequential Organ Failure Assessment) score was used to assess the disease severity of the patient. GCS (Glasgow Coma Scale) marked to analyze the conscious level of the patient, all these assessments were computed for every patient in the hospital during ICU admission and discharge days. On the day of ICU admission and discharge anthropometric indices were collected, for every patient in which height measuring ulna length and weight calculated from the patient's height. BMI was computed by using the formula weight in $\mathrm{kg}$ divided by height in meter square. MUAC (Mid-upper-arm Circumference) and calf circumference were measured as shown in Figure: 1.Public Health sponsored study acknowledged that MUAC has been used for evaluation of adult nutritional status as well, especially in resourcelimited settings, including India. MUAC can be an efficient indicator of adult undernutrition - comparable or even better than BMI (Das et al., 2020).

SGA questionnaire was used for every patient on the date of ICU admission and discharge. SGA is a widely accepted nutritional 


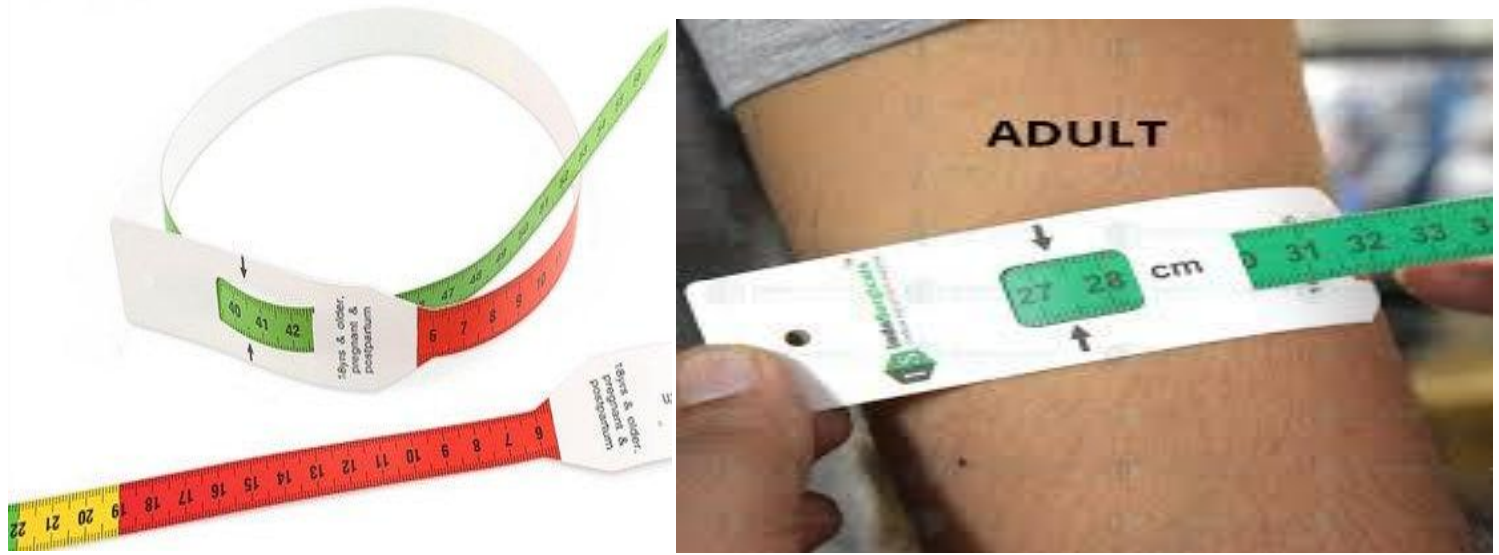

Figure 1 MUAC TAPE Colour Indicator tape

Table 1 BMI and MUAC Comparison

Corresponding BMI category

\begin{tabular}{lll}
\hline$<17 \mathrm{~kg} \mathrm{~m}^{-2}$ & & \\
(moderately $<18.5 \mathrm{~kg} \mathrm{~m}^{-2}$ & $\geq 25 \mathrm{~kg} \mathrm{~m}^{-2} \geq 30 \mathrm{~kg} \mathrm{~m}^{-2}$ \\
underweight) (underweight) & (overweight) & (obese)
\end{tabular}

$\operatorname{MUAC}(\mathrm{cm})$

$\begin{array}{lllll}\text { Men } & 22.9 & <23.9 & \geq 28.1 & \geq 31.4 \\ \text { Women } & 22.9 & <23.9 & \geq 28.4 & \geq 31.8\end{array}$

Source: researchgate.net

assessment tool. It includes details such as weight-loss history, diet intake pattern, GI (gastrointestinal) symptoms, functional capacity, and comorbidity details. This questionnaire also indicates details related to muscle wasting and wastage of subcutaneous fat. All mentioned data were entered to finalize the score as $\mathrm{A}, \mathrm{B}$, or $\mathrm{C}$ an indicator of the degree of malnutrition. Score A indicates well nourished, B denotes mild to moderate malnutrition, and $\mathrm{C}$ indicates severe malnutrition On ICU admission and discharge date biochemical indices such as total protein, albumin, phosphorous, magnesium, calcium, and TLC -total lymphocyte count were collected (Auto analyzer was used).

Important details like ventilator-free days and duration of ICU / hospital stay (in days) was recorded and compared with APACHE IV SCORE (predictors for mortality rate and length of ICU stay).

\subsection{Statistical Analysis}

Statistical package for social sciences was used (SPSS version 19) for statistical analysis. The data were represented as mean and standard deviation. The comparison of data between admission and discharge were done by a paired t-test. The SGA distribution was compared by using the sign test. The duration of ICU/hospital,
Ventilator free days, and malnutrition status analysis related to ICU admission and discharge day was compared by using Pearson's Coefficient correlation $\mathrm{P} \leq 0.05$.

\section{Results}

Complete data collection was carried out for the 325 ICU admitted patients for 3 months, among this finally 120 eligible patients' results were presented in this observational study (Table 2).

In table 2, the Patient's age, sex, and admission category were given, it revealed that the average patient's age was $62.2 \pm 14$ years, among these $55.3 \%$ were males, and the rest $44.7 \%$ were females. Further, among the selected respondents, $52.8 \%$ were admitted for medical management and $47.2 \%$ for surgical intervention.

Results given in table 3 provides patient admission diagnosis among the study population. Among the diagnosis, the highest number of patients was reported for Trauma cases (\%), this was followed by Neurological disorders. While the lowest patients number was reported for Sepsis (5\%), Renal (5\%), and Orthopaedic. Mortality and disease severity score was provided in table 4. The patient's clinical outcomes were shown in this table 5. 
Table 2 Age, Sex \& Admission category of the patients

\begin{tabular}{|cc|}
\hline Variable & $\mathrm{N}, 120 \mathrm{Mean} \pm \mathrm{SD}$ or $\mathrm{n}(\%)$ \\
\hline Average Age (y) & $62.2 \pm 14.1$ \\
\hline Male & $55.3 \%$ \\
\hline Female & $44.7 \%$ \\
\hline & \\
\hline Medical & Admission Category: \\
\hline Surgical & $66(52.8 \%)$ \\
\hline
\end{tabular}

Table 3 Diagnosis of the selected Patient during ICU admission

\begin{tabular}{|cccccc|}
\hline Diagnosis & Patients (\%) & Diagnosis & Patients (\%) & Diagnosis & Patients (\%) \\
\hline Cardiovascular & $12(6.6 \%)$ & Gastrointestinal & $11(9.2 \%)$ & Renal & $6(5 \%)$ \\
\hline Neurological & $22(26.6 \%)$ & Orthopaedic & $2(1.6 \%)$ & Respiratory & $14(11.7 \%)$ \\
\hline Trauma & $40(33 \%)$ & Sepsis & $6(5 \%)$ & Cancer & $7(5.8 \%)$ \\
\hline
\end{tabular}

Table 4 Mortality and Disease severity score

\begin{tabular}{|cc|}
\hline Variable & N, 120Mean \pm SD or n (\%) \\
\hline APACHE ${ }^{\dagger}$ IV score & $70 \pm 21$ \\
\hline Admission SOFA score & $8.4 \pm 2.8$ \\
\hline Admission GCS & $7 \pm 3.2$ \\
\hline
\end{tabular}

Here, APACHE (Acute physiology and chronic health evaluation); SOFA (Sequential organ failure assessment); GCS

(Glasgow coma scale)

Table 5 Clinical and Mortality related patient outcome details

\begin{tabular}{|cc|}
\hline & Mean \pm SD, Median (IQR) \\
\hline Duration of ICU stay (d) & $13(7-22)$ \\
\hline Duration of hospital stay (d) & $20(12-39.5)$ \\
\hline Mortality in ICU (n, \%) & $37(29.6 \%)$ \\
\hline Mortality in hospital (n, \%) & $45(36 \%)$ \\
\hline Duration of ventilator-free days (d) & $4(0.5-10)$ \\
\hline
\end{tabular}

IQR: Interquartile range; SD: Standard deviation; ICU: Intensive care unit

Table 6 Anthropometric Measurements

\begin{tabular}{|cccc|}
\hline Measurements & Admission day & Discharge day & P value \\
\hline Weight $(\mathrm{kg})$ & $63.4 \pm 12.7$ & $56.6 \pm 11.6$ & $<0.001^{\ddagger}$ \\
\hline BMI $\left(\mathrm{kg} / \mathrm{m}^{2}\right)$ & $25.7(23.3-28.8)$ & $22.8(21.3-26.5)$ & 0.088 \\
\hline MUAC $(\mathrm{cm})$ & $30.1 \pm 4.5$ & $27.6 \pm 4$ & $<0.001^{\ddagger}$ \\
\hline Calf circumference $(\mathrm{cm})$ & $32.2 \pm 3.8$ & $29.1 \pm 3.7$ & $<0.001^{\ddagger}$ \\
\hline
\end{tabular}

BMI: Body mass index; MUAC: Mid-upper-arm circumference

Table 7 Subjective Global Assessment Percentage (SGA \%)

\begin{tabular}{|ccc|}
\hline SGA (n, \% $)^{\dagger}$ & Admission day & Discharge day \\
\hline A (Well nourished) & $60(50 \%)$ & $45(37.5 \%)$ \\
\hline B(Moderately malnourished) & $43(35.8 \%)$ & $52(43.3 \%)$ \\
\hline C (Severely malnourished) & $17(14.2 \% \%)$ & $23(19.2 \%)$ \\
\hline
\end{tabular}

SGA: Subjective Global Assessment, 
Table 8 Biochemical parameters on Admission and Discharge Day

\begin{tabular}{|c|c|c|c|}
\hline \multicolumn{2}{|c|}{ Admission day } & Discharge day & 0.968 \\
\hline Total protein $(\mathrm{g} / \mathrm{dl})$ & $6 \pm 1.05$ & $6.2 \pm 1$ & 0.16 \\
\hline Albumin $(\mathrm{g} / \mathrm{dl})$ & $3.4 \pm 0.6$ & $3.3 \pm 0.6$ & 0.146 \\
\hline $\mathrm{TLC}$ & $1242.5(787.6-2099)$ & $279.2(1042-1699)$ & $0.013 \ddagger$ \\
\hline $\mathrm{Mg}(\mathrm{mg} / \mathrm{dl})$ & $2.2 \pm 1.2$ & $3.3 \pm 0.7$ & 0.72 \\
\hline $\mathrm{P}(\mathrm{mg} / \mathrm{dl})$ & $3.5 \pm 1.2$ & $8.5 \pm 0.6$ & 0.43 \\
\hline $\mathrm{Ca}(\mathrm{mg} / \mathrm{dl})$ & $8.4 \pm 0.67$ & & \\
\hline
\end{tabular}

TLC: Total lymphocyte count; Mg: Magnesium; P: Phosphorous; Ca: Calcium

Table 9 SGA status correlation on ICU discharge day, admission day, and outcomes

\begin{tabular}{|ccc|}
\hline Measurements on admission day & Correlation coefficient (Rho Spearman) & P value \\
\hline Weight $(\mathrm{kg})$ & -0.412 & $<0.001^{\dagger}$ \\
\hline BMI $\left(\mathrm{kg} / \mathrm{m}^{2}\right)$ & -0.426 & $<0.001^{\dagger}$ \\
\hline MUAC $(\mathrm{cm})$ & -0.459 & $<0.001^{\dagger}$ \\
\hline Calf circumference $(\mathrm{cm})$ & -0.481 & 0.334 \\
\hline Total protein $(\mathrm{g} / \mathrm{dl})$ & 0.106 & 0.221 \\
\hline Albumin $(\mathrm{g} / \mathrm{dl})$ & 0.132 & 0.244 \\
\hline TLC & -0.127 & 0.622 \\
\hline Mg (meq/l) & -0.059 & 0.852 \\
\hline P (mg/dl) & -0.021 & 0.062 \\
\hline Ca (mg/dl) & -0.179 & $0.002^{\dagger}$ \\
\hline Length of ICU stay (d) & 0.328 & 0.11 \\
\hline Length of ventilator-free days & 0.288 & \\
\hline
\end{tabular}

${ }^{\dagger} \mathrm{P} \leq 0.05$ was considered statistically significant

The mean duration of stay in ICU and hospital was 13 and 20 days respectively. On an average the patient's stay in ICU were 7.9 days and 27.4 days in the hospital. Ventilator free days were noted as 4 days (mean value taken). In addition to this ICU \& hospital mortality percentage was indicated as Mean $29.6 \%$ and $36 \%$ respectively. Anthropometric details collected during admission and discharge days were provided in table 6. SGA on Admission and discharge were shown in table 7. A significant decrease was noted in the Nutritional status of the ICU patients. Nutrition status was inversely proportional with the duration of ICU stay. On ICU discharge day malnutrition scores increased significantly in comparison with admission-day scores. Biochemical parameters recorded on the admission day and discharge day were tabulated in table 8. Except for magnesium, the rest of the data did not change significantly during ICU stay but magnesium level decreased during ICU discharge day. Discharge day malnutrition score and duration of ICU stay showed a positive significant correlation
(Table 9). No significant correlation was noted in the discharge day malnutrition details and ICU ventilator independent days.

\section{Discussion}

Malnutrition in critically ill patients during ICU stay had a strong association with increased risk of mortality and morbidity rate. As per the study, nutrition assessment revealed that the malnutrition percentage of ICU patients on admission day (50\%) had increased significantly on discharge day $(62.5 \%)$. As per the SGA study, ICU patient's malnutrition prevalence on admission day was $50.0 \%$ $(35.8 \%$ \& $14.2 \%$ were mildly-moderately and severely malnourished respectively) which significantly increased on discharge day and it reached $62.5 \%(43.3 \% \& 19.2 \%)$. In the current research, the variation in the nutritional status of the participants is related to age, the previous health status of the patients, and presence of comorbidities, $41.2 \%$ were with a history 
of hypertension and $48.5 \%$ were diabetic and $60.3 \%$ of participant were in the geriatric age group. All these factors like age, comorbidities, and health status hurt their nutritional status. In the present study, malnutrition is strongly associated with a longer duration of ICU/ hospital stay, and it is evident when data were compared between malnourished and well-nourished patients. Medical Nutrition therapy always plays an important role in patient outcomes and recovery. In critical care units the anthropometric measures, MUAC measurements were utilised for assessing adult malnutrition.

This study showed a significantly lower level of values such as mean MUAC, Calf circumference among mild/moderate and severe malnourished patients versus well nourished patients. Accordingly, Haung et al. (2000) a reduction in anthropometric indices during a patient's ICU stay (Mehta et al., 2018).

The patient's detailed assessment depicted that mean weight, calf circumference, and MUAC, had significantly reduced. Similarly, Nematy et al. (2012) findings showed a significant decrease in weight and MUAC readings among critically ill patients. This indicated a higher incidence of malnutrition among ICU patients (Mendes et al., 2017; Vallejo et al., 2017; Wischmeyer, 2017). Besides this study also highlighted a decrease in nutritional status on discharge day (table 7).

Further, the present study also revealed that the serum albumin levels were significantly lower in malnourished patients (mild or severe) when compared with well-nourished groups and this low albumin levels were associated with an extended period of hospital stay. In the present study, the overall mortality rate was $41.2 \%$ whereas mortality rate of $55.9 \%$ and $26.5 \%$ was observed among malnourished and well nourished group respectively which was significantly lower in well nourished participants. So malnutrition is strongly associated with negative outcomes of critically ill patients in the term of increased morbidity and mortality (Nicolo et al., 2016).

Binary logistic regression was performed with lower nutritional status detected by SGA data and it was an independent predictor noted with a negative correlation with mortality. It also clearly indicates the mortality risk was two times higher for a malnourished patient compared to well-nourished participant. The predicted mortality risk scoring - APACHE IV and SOFA scores correlated positively with the observed mortality rate and length of stay in ICU. Bendavid et al. (2017) study result well correlated with the present study that malnourished patient's mortality rate was higher compared with the well-nourished patients (Schiesser et al., 2009; Vallejo et al., 2017). During ICU stay the Biochemical data evaluation revealed a significant decrease in serum magnesium concentration. Hypomagnesaemia was considered as a single indicator of disease severity, morbidity, and mortality in ICU patients (Singer et al., 2019). The low magnesium level was prevalent due to poor nutritional status and low magnesium intake during hospitalisation.

In this study, critically ill patient's anthropometric indices (table 6) were considered as good predictors of nutritional outcomes (malnutrition severity scoring system) compared to patient's biochemical data (Table $8 \& 9$ ). Moreover, albumin level and SGA showed a significant correlation (Table 8). Thus, SGA analysis along with anthropometric parameters was a valuable and effective method in the evaluation of malnutrition status of hospitalised patients, especially for critically ill patients. The study limitation was that heterogeneous ICU patients with different medical or surgical problems were included in the study. However, these different types of ICU patients were intentionally involved in the study because the main aim of the study was to identify the malnutrition prevalence among critically ill hospitalised patients on admission and discharge days without considering the causes of their diseases.

\section{Conclusion}

To conclude, Subjective Global Assessment revealed that malnutrition on ICU discharge day increased to $57.32 \%$. It was highlighted that nutritional assessment with detailed anthropometric details should be collected and SGA to be done for every patient entering into ICU. This initial approach paved the way to implement the timely nutritional intervention, and it reduced nutrition-related complications and resulted in an appropriate Nutrition care process.

The study clearly stated that prioritizing nutritional assessment early at ICU admission paved the way to the appropriate nutritional plans which minimize the impact of malnutrition on the patient's outcome and it helped to reduce the duration of ICU and hospital stay. SGA, timely nutrition intervention and medical nutrition therapy prevent hospital-acquired infection and thereby mortality rate reduced. The nutrition support team and Nutrition protocol implementation in all ICU is crucial and it is very important to achieve a positive outcome for a critically ill patient.

\section{Acknowledgment}

This study was extracted from an on-going Ph.D. thesis Nutrition Assessment and Specialized Nutrition Therapy for Critically Ill Patients. The authors would like to thank the Madurai Kamaraj University for their guidance and support in conducting the study.

Conflict of Interest: None declared. 


\section{References}

Bendavid I, Singer P, Thellia M (2017) Nutrition Day ICU: A 7 year worldwide prevalence study of nutrition practice in intensive care. Clinical Nutrition 36:1122-9.

Blaser AR, Starkop FJ, Alhazzani W (2017) Early enteral nutrition in critically ill patients: ESICM clinical practice guidelines. Intensive Care Medicine 43:380-98.

Chakravarty C, Hazarika B, Goswami L, Ramasubban S (2013) Prevalence of malnutrition in a tertiary care hospital in India. Indian Journal of Critical Care Medicine17:170-3.

Das A, Saimala G, Reddy N, Mishra P, Giri R, Kumar A, Raj A, Kumar G, Chaturvedi S, Babu S, Srikantiah S, Mahapatra T (2020) Mid-upper arm circumference as a substitute of the body mass index for assessment of nutritional status among adult andadolescent females: learning from an impoverished Indian state. Public Health 179: 68-75.

Haung YC, Yen CE, Cheng CH, JihksKan MN (2000) Nutritional status of mechanically ventilated critically ill patients: comparison of different types of nutritional support. Clinical Nutrition 19:101 107.

Herridge MS, Moss M, Hough CL, Hopkins RO, Rice TW, Bienvenu OJ, Azoulay E (2016) Recovery and outcomes after the acute respiratory distress syndrome (ARDS) in patients and their family caregivers. Intensive Care Medicine 42(5):725-38.

Heyland DK, Dhaliwal R, Jiang X, Day AG (2011) Identifying critically ill patients who benefit the most from nutrition therapy: The development and initial validation of a novel risk assessment tool. Critical Care 15:R268.

Hopkins RO, Suchyta MR, Kamdar BB, Darowski E, Jackson JC, Needham DM (2017) Instrumental activities of daily living after critical illness: a systematic review. The Annals of the American Thoracic Society 14(8):1332-43.

Lew CC, Yandell R, Fraser RJ, Chua AP, Chong MF, Miller M (2017) Association between malnutrition and clinical outcomes in the Intensive Care Unit: A Systematic review. JPEN Journal of Parenteral and Enteral Nutrition 41:744-58.

McClave SA, Taylor BE, Martindale RG, Warren MM, Johnson DR, Braunschweig C (2016) Guidelines for the provision and assessment of nutrition support therapy in the adult critically ill patient: Society of Critical care Medicine (SCCM) and American Society for Parenteral and Enteral Nutrition (A.S.P.E.N.). JPEN Journal of Parenteral Enteral Nutrition40:159-211.

Mehta Y, Sunavala JD, Zirpe K, Tyagi N, Garg S, Sinha S (2018) Practice guidelines for nutrition in critically ill patients: A relook for Indian scenario. Indian Journal of Critical Care Medicine 22:263-73.

Mendes R, Policarpo S, Fortuna P, Alves M, Virella D, Heyland DK (2017) Nutritional risk assessment and cultural validation of the modified NUTRIC score in critically ill patients-A multicenter prospective cohort study. Journal of Critical Care 37: 249.

Nicolo M, Heyland DK, Chittams J, Sammarco T, Compher C (2016) Clinical outcomes related to protein delivery in a critically ill population: a multicenter, multinational observation study. JPEN Journal of Parenteral and Enteral Nutrition 40(1):45-51.

Schiesser M, Kirchhoff P, Müller MK, Schäfer M, Clavien PA (2009) The correlation of nutrition risk index, nutrition risk score, and bioimpedance analysis with postoperative complications in patients undergoing gastrointestinal surgery. Surgery145: 519-526.

Simon Lambden, Pierre Francois Lattere, Mitchell M Levy, Bruno Francois (2019) The SOFA score - development, utility and challenges of accurate assessment in clinical trials. Critical care 23, Article number :374

Singer P, Blaser AR, Berger MM, Alhazzani W, Calder PC, Casaer MP (2019) ESPEN guideline on clinical nutrition in the intensive care unit. Clinical Nutrition38(1):48-79.

Vallejo KP, Martinez CM, Adames AM (2017) Current clinical nutrition practices in critically ill patients in Latin America: a multinational observational study. Critical Care 21:227.

Wischmeyer PE (2017) Tailoring nutrition therapy to illness and recovery. Critical Care 21(3):316.

Nematy M, Mohajeri SAR, Moghadam SA, Safarian M, Norouzy A, Parizadeh SMR, et al. Nutritional status in intensive care unit patients: a prospective clinical cohort pilot study. Mediterranean Journal of Nutrition and Metabolism 5:163-8. doi: 10.1007/s12349-011-0071-x 